\title{
MicroRNA-21 Promotes Allergic Airway Inflammation and AHR and Inhibits Mesenchymal Stem Cell Migration in Cockroach Allergen Induced Asthma Model
}

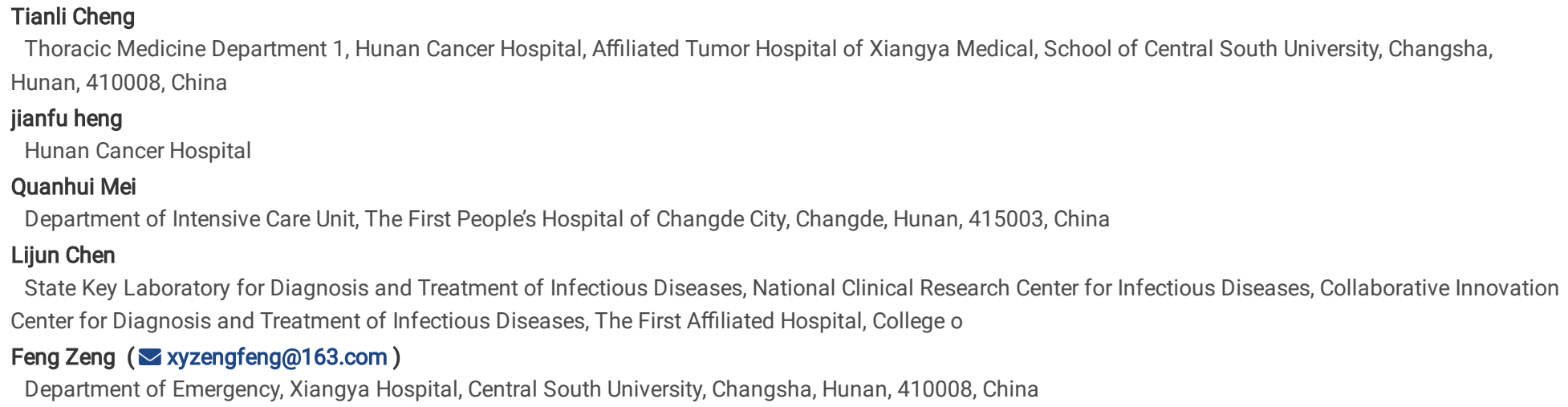




\section{Abstract}

Background

Mesenchymal stem cells (MSCs) have been used to treat asthma in a mouse model. However, the efficacy and mechanism of MSCs are not elucidated. MicroRNAs (miRNAs) play a key rolein asthma and related to the aim of this study was to illustrate the role of miR21 and its influence on MSC migration in asthma model.

Methods

A mouse model of asthma was established using cockroach extract (CRE), and miR-21 expression was examined. A miR-21 lentivirus construct was used to investigate the role of miR-21 in vivo and in vitro in mouse bone marrow-derived (BM-) MSCs. A TOPFlash reporter gene assay was used to study the signaling downstream of miR-21. IL-4, IL-5, IL-13, IgE, and IgG1 levels in bronchoalveolar lavage fluids were determined by enzyme-linked immunosorbent assays.

Results

MiR-21 was upregulated in CRE-induced asthmatic mice. MiR-21 promoted allergic airway inflammation and airway hyperreactivity by inhibiting BM-MSC migration. $\beta$-Catenin was found to act downstream of miR-21 as a negative regulator of miR-21. Rescue experiments verified that miR-21 inhibited BM-MSC migration by suppressing $W n t / \beta$-catenin signaling.

Conclusion

MiR-21 promotes allergic airway inflammation and AHR and inhibits BM-MSC migration through Wnt/ $\beta$-catenin signaling, which may serve as an effective therapeutic target for asthma.

\section{Introduction}

Asthma is a chronic inflammatory disorder characterized by airway obstruction, heterogeneous inflammation, mucus secretion, airway hyperresponsiveness (AHR), and high expression of immunoglobulin E (IgE) [1-4]. Various behavioral activities, environmental factors, stimulating allergies, and excessive T-helper type 2 ( $\mathrm{TH} 2)$ cell responses are considered major causes of asthma $[5,6]$. Currently, the treatment of asthma mainly depends on allergen immunotherapy targeting allergic immunocytes including TH2 cells, B cells, T cells, B regulatory cells, T regulatory cells, mast cells, macrophages, and dendritic cells [7-10]. Due to diverse pathogens and evolutionary antigens, the body produces stronger resistance, and some steroids are being developed for the treatment of asthma [11]. Although current drugs and allergen immunotherapies are effective to a certain extent, they have numerous side effects, including decreased bone mineral density, skin thinning and bruising, immunologic tolerance, rejection, and anaphylaxis [12, 13]. And the morbidity of asthma is still increasing [14, 15]. Therefore, novel therapeutic strategies targeting pathophysiological events need to be further explored.

Mesenchymal stem cells (MSCs) have attracted considerable attention for the treatment of various diseases, both in basic medicine and pre-clinical research, owing to their self-renewing and differentiating properties [16-23]. Murine bone marrow-derived (BM-) MSCs inhibited allergic responses in a mouse model of asthma [24], and human BM-MSCs suppressed chronic airway inflammation in a murine asthma model [25]. Increasing studies have shown that MSCs ameliorate asthma in both mouse models, and the mechanisms underlying the therapeutic effects of MSCs have been explored [26-30]. However, little is known about the mechanism underlying MSC migration to target sites to exert curative effects. Migration is one of the vital characteristics of MSCs and is crucial for MSC therapy targeting inflammatory sites. It is fundamental to the application of MSC-based asthma treatments in future clinical research.

MicroRNAs (miRs) are small, non-coding RNAs that play roles in transcriptional and post-transcriptional gene expression regulation by targeting the 3'untranslated regions of specific mRNAs [31-33]. Recent evidence suggests that miRs play a critical role in the diagnosis and treatment of asthma [34-36]. Accumulating evidence indicates that MSCs ameliorate asthma via miRs that mediate relevant signaling pathways [37-40]. Additionally, some studies have shown interactions between MSCs and miRs in asthma [37, 41, 42]. MiR-21, which is related to cell apoptosis, invasion, and migration, is one of the most widely researched miRs $[43,44]$. It has been reported to suppress tumor cell migration into injured areas in several cancers [44-46]. Based on a literature review, we found that miR-21 is mostly overexpressed in asthma [1-3], which we validated in preliminary experiments. Therefore, we aimed to investigate the role and mechanism of miR-21 in asthma treatment with MSCs.

In this study, the miR-21 expression and its influence on allergic airway inflammation and AHR and MSC migration were assessed in cockroach extract (CRE)induced mouse model of asthma. MiR-21 overexpression and silencing were used to evaluate the signaling pathway mediating MSC migration in vivo and in vitro.

\section{Materials And Methods}

\subsection{Asthma mouse model establishment}

Six- to eight-week-old C57BL/6 mice were purchased from SLAC Laboratories (Shanghai, China). The mice were housed under standard conditions with a 12h/12-h light/dark cycle at the Animal Center of Hunan Cancer Hospital (Changsha, China) and had free access to food and water. All animal experiments and procedures were performed according to the guidelines of the Center for Medical Ethics, Central South University.

A German CRE (B46, Blattella germanica)-induced asthma mouse model was conducted. In brief, mice were sensitized by intratracheal inhalation of $20 \mu \mathrm{g}$ CRE in $50 \mu \mathrm{L}$ of PBS under anesthesia using isoflurane (Sigma, USA) on days (D) $0-4$. The mice were challenged by intratracheal instillation of the same 
amount of CRE on D10-D13. Control mice received the same volume of PBS during the sensitization and challenge phases. Mice were randomly assigned to the two treatment groups $(n=6$ each) and were analyzed in a double-blind manner. On D14, the mice were sacrificed. The lung tissues were dissected for histological analyses, and bronchoalveolar lavage fluids (BALFs) were harvested to count total cells and inflammatory cells. A timeline of the mouse experiment is shown in Figure 1A.

\subsection{Analysis of lung inflammation}

Lung inflammation was assessed. Briefly, BALFs were centrifuged at $300 . g$ at $4^{\circ} \mathrm{C}$ for $10 \mathrm{~min}$ and washed with ice-cold PBS. Total cells and inflammatory cells including eosinophils (Eos), lymphocytes (Lym), macrophages (Mac), and neutrophils (Neu), were counted to evaluate the percentages of inflammatory cells in BALF by flow cytometry using a FACS Calibur cytometer (BD Biosystems).

IL-4, IL-5, and IL-13 in mouse BALFs were examined by ELISAs, using commercial kits (eBioscience, USA) according to the manufacturer's recommendations. Serum CRE-specific IgE and IgG1 were examined by ELISAs.

\subsection{Cell cultureand lentivirus infection}

MSCs were cultured in a-minimum Eagle's medium (Hyclone, USA) supplemented with $10 \%$ fetal calf serum (Hyclone), $100 \mathrm{mg} / \mathrm{mL}$ streptomycin (Gibco, USA), and $100 \mathrm{IU} / \mathrm{mL}$ penicillin (Gibco) in a humidified incubator with $5 \% \mathrm{CO}_{2}$ at $37^{\circ} \mathrm{C}$ (Thermo Fisher, USA). Cells were passaged in tissue-culture flasks (Corning, USA) in medium containing $0.25 \%$ trypsin-EDTA (Gibco) until approximately $80 \%$ confluence. Detailed cell-culture procedures were reported in our previous study [47]. MSCs at passages below 8 were used in all experiments.

MiR-21, anti-miR-21, miR negative control (NC), and anti-miR-NC oligonucleotides (50 nM, RiboBio, Guangzhou, China) were transfected into MSCs using the riboFECT ${ }^{\text {TM }}$ CP Transfection Kit (Ribo) according to the manufacturer's protocol. The sequence of Pri-miR-21 was as follows: 5'-

TCACAAGACATAAGGACCACAATTTTTGACTGCAAACCATGATGCTGGGTAATGTTTGAATGAAAACATTTGATATGG ATGGTCAGATGAAAGATACCAAAATGTCAGACAGCCCATCGACTGCTGTTGCCATGAGATTCAACAGTCAACATCAGTCTGATAAGCTATCCGACAAGGTGGTACAGCCA7 ATGTCACGACCACGACAGGGGGCAAGCACGAGGTGCTCAGGCAGGGTTTAAAGCAAAGCAAACATCTCTGGTTTGCTGGGGCTT-3'. The miR-21 sequence was 5'GTGCATTGCTGTTGCATTGC-3'. BM-MSCs were seeded in a six-well plate (Corning) at $1 \times 10^{5}$ cells/well and infected with lentiviral anti-miR-21 and miR-21 mimic, and miR-NC and anti-miR-NC as controls, in the presence of $10 \mathrm{mg} / \mathrm{mL}$ polybrene (Millipore, Germany).

Small interfering (si) RNA targeting the $\beta$-catenin gene (Ctnnb1) was synthesized at GenePharma (Shanghai, China). An unrelated sequence was used as a negative control, according to our previous report [48]. Briefly, siRNAs were transfected into BM-MSCs during the logarithmic growth phase using Lipofectamine 2000 (Invitrogen, USA) according to the manufacturer's instructions. During the challenge period (D10-13), mice were intranasally administrated $2 \times 10^{6}$ infectious units (IFUs) of miR-21 lentivirus, and an equal amount of empty lentiviral vector was used as a control.

\subsection{BM-MSC migration assays in vivo and in vitro}

To verify whether exogenous BM-MSCs can migrate to the lungs in asthmatic mice, $5 \times 10^{6} \mathrm{GFP}^{+} \mathrm{BM}-\mathrm{MSC}$ in $0.2 \mathrm{~mL}$ of PBS were injected via the tail vein, and the same amount of PBS was injected as a control. To assay MSC migration in vitro, BM-MSCs $\left(1 \times 10^{5}\right)$ were transfected with a plasmid expressing miR21, anti-miR-21, and $\beta$-catenin using Lipofectamine 2000 (Invitrogen) in serum-free minimum Eagle's medium at $37^{\circ} \mathrm{C}$ in a $5 \% \mathrm{CO}_{2}$ atmosphere for $48 \mathrm{~h}$. Then, the cells were added to the upper chambers of Transwell inserts with 8.0- $\mu \mathrm{m}$ pores in serum-free medium, while normal growth medium was placed in the lower chambers. After incubation for $24 \mathrm{~h}$, the MSCs were fixed and stained with $20 \%$ methanol violet (Beyotime, China) and $0.1 \%$ crystal violet (Beyotime, China), and counted under a microscope.

\subsection{Quantitative reverse-transcription (q-RT) PCR}

To evaluate the expression of miR-21 and Ctnnb1 in BM-MSCs, total RNA was isolated from BM-MSCs using TRIzol reagent (Invitrogen, USA). The RNA was treated with RNase-free DNase I (Roche, Basel, Switzerland). cDNA was synthesized using the All-in-One ${ }^{\text {TM }}$ First Strand cDNA Synthesis Kit (AORT-0050, Genecopoeia, USA) and miRNA First Strand cDNA Synthesis Kit (AMRT-0050, Genecopoeia). RT-qPCRs were run using the All-in-One ${ }^{\text {TM }}$ qPCR mix (Genecopoeia) and All-in-One ${ }^{\mathrm{TM}}$ miRNA qRT-PCR Detection Kit (Genecopoeia) on an ABI 7300HT real-time PCR system (Applied Biosystems, USA). Primers for miR-21, Ctnnb1, Gapdh, and U6 were synthesized at Genecopoeia. Relative expression levels of miR-21 and Ctnnb1 were evaluated using the $2^{-\Delta \Delta C T}$ method $[49,50]$. U6 was used as an endogenous control for miR-21, and Gapdh was used as an internal control for Ctnnb1.

\subsection{Westem blotting}

Lung tissues and BM-MSCs from the different treatment groups were collected and homogenized in RIPA lysis buffer (Beyotime, China) supplemented with 1 $\mathrm{mM}$ phenylmethylsulfonyl fluoride (Beyotime). Protein concentrations were determined using a BCA Protein Assay Kit (Beyotime), and $25 \mu \mathrm{g}$ protein was loaded per lane. Western blotting was conducted as described previously [48]. The primary antibodies used were anti- $\beta$-catenin (D10A8, CST, 1: 1000) and antiGAPDH (14C10, CST, 1: 1000), and goat anti-rabbit IgG (Invitrogen) was used as the secondary antibody. Immunoreactive bands were visualized with enhanced chemiluminescence reagent (Bio-Rad, USA), using a Tanon-4500 digital image system (Tanon Science \& Technology, China).

\subsection{Immunostaining and immunofluorescence}

For the histological assessment of lung inflammation, mouse lungs were collected and soaked in $10 \mathrm{~mL}$ of ice-cold PBS. The samples were fixed in $4 \%$

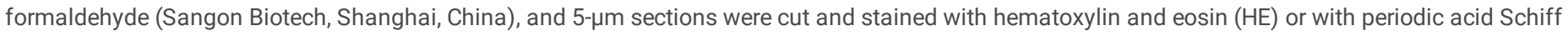


reagent (Sigma). The sections were examined and photographed under a microscope (Nikon, Japan). Detailed immunostaining procedures for lung histology were described in our previous report [48].

For immunofluorescence, lung sections were blocked with protein-blocking serum-free solution (Dako, Denmark), permeabilized with $0.1 \%$ Triton $\mathrm{X}-100$ for 10 min, and blocked in $1 \% \mathrm{BSA}$ at room temperature for $30 \mathrm{~min}$. The sections were incubated with primary antibody against $\beta$-catenin (1:100 dilution) at $4{ }^{\circ} \mathrm{C}$ overnight. The sections were washed and incubated with specific secondary antibody in PBS (1:100 dilution) for 60 min. Nuclei were stained with DAPI (Beyotime) at room temperature for $10 \mathrm{~min}$. Immunofluorescence was evaluated under a confocal microscope (Leica Microsystems, Germany). The intensity of co-staining was determined using image acquisition and analysis software (Image $\mathrm{J}$ ), and the values are presented as mean fluorescence intensity per square micro.

\subsection{TOPFlash reporter gene assay}

To evaluate the signaling downstream of miR-21, we used a pair of TOPFlash/FOPFlash luciferase reporter constructs (Upstate Biotechnology, USA). BMMSCs were seeded in a 24-well plate (Corning) at $1 \times 10^{5}$ cells/well and allowed to settle for $24 \mathrm{~h}$. TOPFlash or FOPFlash plasmid (500 ng), pRL-TK Renilla luciferase plasmid (150 ng; Promega, USA), and miR-21 mimic ( $50 \mathrm{nM})$ or miR-NC ( $50 \mathrm{nM}$ ) were cotransfected into the BM-MSCs. After $24 \mathrm{~h}$ of incubation, the cells were stimulated with $50 \mathrm{ng} / \mathrm{mL}$ recombinant murine Wnt3a (PeproTech, USA) for another $24 \mathrm{~h}$. Then, the cells were harvested and luciferase reporter activity was measured in the reporter lysis buffer of the Luciferase Assay System (Promega). TOPFlash and FOPFlash signals were normalized to Renilla luciferase activity, and data are represented as normalized TOPFlash/FOPFlash activity values.

\subsection{Statistical analysis}

Data are reported as the mean \pm SD derived from at least three independent experiments. Means were compared using a two-tailed paired Student's $t$-test or using the Chi-square test for categorical variables. $P<0.05$ was considered statistically significant.

\section{Results}

\subsection{MiR-21 expression is increased in CRE-induced asthma model mice}

MiR-21 expression was significantly higher in CRE-challenged mice than in PBS-treated mice (Figure 1B). Further, miR-21 was significantly upregulated in miR21-transfected CRE model mice as compared to miR-NC-transfected mice (Figure 1C).

\subsection{MiR-21 promotes lung inflammation in asthma}

To understand the role of miR-21 in asthma, we conducted histological analysis. HE staining demonstrated that miR-21 accelerated inflammatory cell infiltration, goblet cell hyperplasia, and mucus overproduction (Figure 2A). Although the numbers of Lym, Mac, and Neu were not significantly different, there were significant increases in total cells and Eos in the miR-21+CRE group when compared with the miR-NC+CRE group (Figure 2B and 2C). The ability of AHR to resist methacholine at $10 \mathrm{mg} / \mathrm{mL}$ and $30 \mathrm{mg} / \mathrm{mL}$ was increased in CRE-challenged miR-21 mice when compared to CRE-challenged miR-NC mice (Figure 2D). Additionally, allergen-specific IgE (Figure 2E) and IgG1 (Figure 2F) levels were significantly increased in CRE-challenged miR-21 mice. Th2-secreted cytokines, including IL-4 (Figure 2G), IL-5 (Figure 2H), and IL-13 (Figure 2I), in BALFs were increased in CRE-challenged miR-21 mice. These results indicated that the aggravated Th2-dependent inflammatory reaction in asthma is related to miR-21 expression.

\subsection{BM-MSCs improve allergic airway inflammation and AHR, whereas miR-21 aggravates inflammatory reactions by suppressing BM-MSC migration}

A schematic diagram of MSC administration in the CRE-induced mouse model was shown in Figure 3A. In miR-21-transfected asthmatic mice, GFP ${ }^{+}$BM-MSCs migrated into the lungs, but the number of BM-MSCs in the lungs was significantly lower than that in miR-NC-transfected asthmatic mice, indicating that miR21 inhibits BM-MSC migration into the lungs (Figure 3B). Mean fluorescence intensities are shown in Figure S1. Next, we aimed to confirm that miR-21 promotes allergic airway inflammation and AHR by inhibiting BM-MSC migration. MiR-21-transfected asthmatic mice engrafted with GFP ${ }^{+}$MSCs exhibited decreased airway inflammation cell infiltration, and goblet cell secretion when compared with miR-21-transfected asthmatic mice administered PBS, suggesting that BM-MSCs inhibit airway inflammation and infiltration (Figure $3 \mathrm{C}$ ). These results were consistent with those of previous studies showing that MSCs suppress chronic/allergic airway inflammation in a murine asthma model [25,27]. Interestingly, inflammatory cell infiltration in the lungs was significantly decreased in the miR-21 + CRE + BM-MSCs group when compared with the miR-21 + CRE + PBS group (Figure 3C). Although the numbers of Lym, Mac, and Neu showed no obvious differences, there were significant decreases in total cells and Eos cells after BM-MSC transplantation in asthmatic mice, whereas the numbers of total cells and Eos in the BALF were significantly increased in the miR-21-transfected group post BM-MSC injection (Figure 3D and 3E). Furthermore, IgE (Figure 3F) and IgG1 (Figure 3G) levels were significantly decreased after BM-MSC transplantation in asthmatic mice, whereas these levels were enhanced in miR-21-transfected mice. IL-4 (Figure 3H), IL-5 (Figure 3I), and IL-13 (Figure 3J) levels in the BALF were significantly decreased after BM-MSC transplantation in asthmatic mice, but were significantly increased in mice transfected with miR-21. These results were consistent with the histological examination findings. Thus, these results corroborated that BM-MSC transplantation significantly improved the inflammatory reaction, whereas miR-21 aggravated the inflammatory reaction by inhibiting BM-MSC migration in asthma.

\subsection{MiR-21 suppresses BM-MSC migration in vitro}

MiR-21 expression in the different treatment groups was confirmed by RT-qPCR (Figure 4A). First, we conducted wound-healing assays to evaluate the effect of miR-21 on the migration ability of BM-MSCs in vitro. Compared with the miR-NC group, BM-MSC migration was suppressed in the miR-21 group at $24 \mathrm{~h}$ and $48 \mathrm{~h}$ (Figure 4B), and when anti-miR-21 was added, BM-MSC migration was obviously enhanced. These results indicated that miR-21 inhibits BM-MSC 
migration in vitro. To assess the role of miR-21 in directed migration of BM-MSCs, we used a Boyden chamber to evaluate BM-MSC migration. As shown in Figure 4C, miR-21 suppressed BM-MSC migration, and the migration ability of BM-MSCs was significantly enhanced when anti-miR-21 was added. Detailed MSC numbers are shown in Figure 4D. Together, these data indicated that miR-21 suppresses BM-MSC migration in vitro.

\subsection{MiR-21 inhibits Wnt/ $\beta$-catenin signaling in BM-MSCs}

To explore the molecular mechanism underlying miR-21-mediated blockade of BM-MSC migration, we investigated the involvement of the Wnt/ $\beta$-catenin pathway. We found that Wnt3a was activated as indicated by increased TOPFlash signals ( 4 -fold) in miR-NC-transfected BM-MSCs, suggesting that Wnt/ $\beta$ catenin signaling was induced (Figure 5A). When BM-MSCs were treated with the miR-21 mimic, Wnt3a was unable to significantly increase TOPFlash activity, suggesting that miR-21 inhibits Wnt/ $\beta$-catenin signaling in BM-MSCs (Figure 5A). Furthermore, overexpression of miR-21 significantly decreased Ctnnb1 expression, whereas knockdown of miR-21 significantly increased Ctnnb1 expression (Figure 5B). Similar results were obtained at the protein level (Figure 5C,

5D). These results indicated that miR-21 inhibits the Wnt/ $\beta$-catenin pathway in BM-MSCs.

\subsection{MiR-21 suppresses Wnt/ $\beta$-catenin signaling to inhibit BM-MSC migration in vitro}

To explore the correlation between BM-MSC migration and Wnt/ $\beta$-catenin signaling further, we assessed the role of $\beta$-catenin in anti-miR-21-transfected BMMSCs. We transfected BM-MSCs with $\beta$-catenin siRNA or siRNA-NC and anti-miR-21 and then measured $\beta$-catenin expression. $\beta$-Catenin protein expression was significantly increased after anti-miR-21 transfection (Figure 6A, 6B). Further, knockdown of $\beta$-catenin using siRNA significantly decreased BM-MSC migration, whereas anti-miR-21 promoted BM-MSC migration by upregulating $\beta$-catenin expression (Figure $6 \mathrm{C}$ ). Additionally, cell staining revealed that knockdown of $\beta$-catenin reduced BM-MSC migration upon addition of anti-miR-21, whereas BM-MSC migration was enhanced by upregulating $\beta$-catenin expression (Figure 6D and 6E). These findings suggested that miR-21 downregulates Wnt/ $\beta$-catenin and subsequently impairs BM-MSC migration in vitro.

\subsection{MiR-21 inhibits BM-MSCs migration by suppressing Wnt/ $\beta$-catenin signaling in vivo}

After demonstrating the role of the Wnt/ $\beta$-catenin pathway in the effect of miR-21 in suppressing BM-MSC migration in vitro, we further assessed the effects of miR-21 on BM-MSC migration in vivo. We found that Ctnnb1 mRNA expression was enhanced after BM-MSC transplantation in asthmatic mice when compared with the PBS control group, and it was obviously decreased in the miR-21 + CRE + BM-MSCs group as compared to the miR-NC + CRE + BM-MSCs group (Figure 3A and 7A), suggesting that miR-21 negatively regulates BM-MSCs in asthma. Similar findings were obtained at the protein level (Figure 7B, 7C, and 7D). Collectively, these results indicated that miR-21 inhibits BM-MSC migration by suppressing Wnt/ $\beta$-catenin signaling in CRE-induced asthma in vivo.

\section{Discussion}

Accumulating evidence demonstrates that transplanted MSCs can repair lung injury and improve inflammatory airway disorders [26, 27, 51]. Recently, researchers have found that miRNAs are involved in airway allergic inflammation in a mouse model of asthma after BM-MSC transplantation [37]. These findings suggested that miRNAs may participate in MSC migration by targeting $\beta$-catenin signaling. In support of this hypothesis, we found that: (1) miR-21 promoted asthma by suppressing MSC migration in a CRE-induced asthma mouse model; (2) miR-21 and anti-miR-21 transfection indicated that miR-21 inhibited BM-MSC migration in vitro; (3) miR-21 inhibited BM-MSC migration by suppressing Wnt/ $\beta$-catenin signaling both in vivo and in vitro. A summarizing diagram of the role of miR-21 in suppressing MSC migration by decreasing Wnt/ $\beta$-catenin signaling in asthma is shown in Figure 8 . We found that miR-21 is involved in the regulation of $\beta$-catenin expression; however, the exact mechanism requires further study. The conventional mechanism of miRNA regulation of gene expression is the inhibition of translation. Sometimes, miRNAs regulate gene expression at both transcriptional and translational levels; in this way, both mRNA and protein expression will be inhibited. In addition, one miRNA can regulate multiple target genes, and target genes of other miRNAs form a complex regulatory network that is extensively involved in biological abnormalities [52]. Collectively, our findings provide a novel insight into the molecular mechanisms underlying the regulation of MSC migration and provide a new therapeutic target for treating asthma.

Several miRNAs are implicated in asthma pathogenesis [34,53], and miRNA-21 is a key factor in allergic airway diseases [44, 45, 54]. Eosinophilic inflammation and IL-4 levels reportedly are reduced and accompanied by an increase in IFN-y levels in ovalbumin-induced airway inflammation in miR-21knockout mice [55]. Further, miR-21 downregulates the expression of phosphatase and tensin homolog, which antagonizes phosphoinositol 3-kinase (PI3K) activity in a severe steroid-insensitive asthma mouse model [43]. Kim et al. showed that treatment with a miR-21-specific antagomir or the PI3K inhibitor LY294002 reduced PI3K activity and restored HDAC2 levels, which led to inhibition of the AHR and restoration of steroid sensitivity in allergic airway disease, indicating that miR-21 is a novel therapeutic target for asthma [44]. More recently, Lee et al. found that inhibition of miR-21 ameliorated allergic inflammation in a mouse model of asthma [45]. In line herewith, we found that suppression of miR-21 can ameliorate allergic inflammation in asthma by reducing the infiltration of inflammatory cells, especially eosinophils, Th2 cytokines IL-4, IL-5, and IL-13 in the BALF, AHR, and specific IgE production in the BALF.

MSCs have shown a therapeutic effect in models of acute lung inflammation and fibrosis by targeting several inflammatory cells, including mast cells, natural killer cells, B cells, T cells, T regulatory cells, and dendritic cells $[56,57]$. MiRNAs can serve as downstream adapters to regulate MSC migration and mediate different diseases [26, 37]. Yue et al. found that miR-124 expression is markedly decreased in MSCs in response to hepatocyte growth factor stimulation via suppression of $\beta$-catenin signaling [58]. Lee et al. reported that miR-543 and miR-590-3p decrease AIMP3/P18 expression levels by modulating cellular aging in MSCs in vitro [42]. More recently, Qiu et al. demonstrated that miR-155 regulates oxidative stress and cyclooxygenase-2 in CRE-induced murine asthma [38]. Thus, several miRNAs can mediate MSCs to influence asthma development and may serve as therapeutic targets.

It is well known that MSC migration into the injured site is crucial for MSC therapy in various diseases. How exactly miR-21 regulates the inflammatory response to exacerbate asthma is still unknown. In the present study, we found that miR-21 expression was markedly increased in the asthma model, and increased miR-21 expression was associated with suppressed MSC migration. MiR-21 could suppress MSC immigration both in vivo and in vitro. Our results 
strongly suggest that miR-21 promotes CRE-induced asthma by suppressing MSC migration. Therefore, miR-21 negatively regulates the chemotactic migration of BM-MSCs, which can serve as a potential target for treating asthma in future.

Wnt/ $\beta$-catenin plays a key role in the development of airway inflammation. Activation of $\beta$-catenin signaling by both Wnt-dependent and Wnt-independent pathways has been demonstrated to contribute to airway remodeling [59]. Wnt/ $\beta$-catenin signaling activation by Wnt3a or LiCl improves the migratory capacity of MSCs [60], and the Wnt inhibitor Dkk-1 promotes pathological Th2-mediated inflammation [61]. Activation of Wnt/ $\beta$-catenin signaling in alveolar epithelial cells attenuates intercellular adhesion molecule 1/vascular cell adhesion molecule 1-mediated adhesion and inhibits lung inflammation [62]. The Wnt/ $\beta$-catenin pathway is one of the major pathways associated with MSC migration [60]. The Wnt pathway is activated in inflammatory bowel disease and can be suppressed by the transplantation of MSCs, which differentiate into intestinal epithelium [63]. MiR-124 expression is markedly decreased in MSCs in response to hepatocyte growth factor stimulation [58]. It seems that Wnt/ $\beta$-catenin signaling plays different roles in inflammatory diseases by targeting inflammatory cells and the immune microenvironment. In the present study, miR-21 was characterized as a novel regulator of the canonical Wnt/ $\beta$-catenin signaling pathway, which is involved in the migration of MSCs. Based on our findings, we conclude that miR-21 inhibits MSC migration by targeting Wnt/ $\beta$ catenin signaling. This conclusion was supported by the following evidence. First, miR-21-treated CRE-induced asthma model mice showed decreased $\beta$ catenin expression compared to miR-NC-treated mice. Second, miR-21-treated asthmatic mice showed less MSC migration than miR-NC-treated mice. Finally, miR-21 was found to be associated with $\beta$-catenin expression in influencing MSC migration both in vivo and in vitro. Taken together, our data indicate that miR-21 inhibits MSC migration by suppressing Wnt/ $\beta$-catenin signaling.

\section{Conclusions}

Our study demonstrated that miR-21 significantly stimulates CRE-induced asthma by inhibiting MSC chemotactic migration both in vivo and in vitro. More importantly, we provided evidence that Wnt/ $\beta$-catenin signaling is involved in the inhibitory effect of miR-21 on the migration of BM-MSCs. MiR-21 facilitates the migration of BM-MSCs, which is a potential target for MSC-based treatment of asthma. Other possible mechanisms involved in MSC migration with regard to the treatment of asthma remain to be explored.

\section{Abbreviations}

AHR: airway hyperresponsiveness; Ig: immunoglobulin; TH2: T helper type 2; IL: interleukin; MSC: mesenchymal stem cell; BM: bone marrow; WT: wild-type; CRE: cockroach extract; BALF: bronchoalveolar lavage fluid; NC: negative control; siRNA: small interfering RNA; IFU: infectious unit; Eos: eosinophil; Lym: lymphocyte; Mac: macrophage; Neu: neutrophile; ELISA: enzyme-linked immunosorbent assay; HE: hematoxylin and eosin; PI3K: phosphoinositol 3-kinase

\section{Declarations}

\section{Funding}

This study was supported by grants from the National Natural Science Foundation of Hunan Province (2020JJ4418 and 2020JJ5793), the Natural Science Foundation of Hunan Province National Health Commission (20201286), and the National Natural Science Foundation of China (81900563).

\section{Ethics approval and consent to participate}

This study was submitted to and approved by the Center for Medical Ethics, Central South University. All protocols were approved by the Institutional Review Board of the Animal Center of Central South University.

\section{Consent for publication}

We confirm that the figures in the manuscript are original, and all authors agreed with publication.

\section{Competing interests}

The author declares no competing financial interests.

\section{Acknowledgements}

The authors thank Prof. Nong Yang from Lung Cancer and Gastroenterology Department, Hunan Cancer Hospital, Affiliated Tumor Hospital of Xiangya Medical School of Central South University for technical help of this manuscript. Additionally, we would like to thank Editage (www.editage.cn) for English language editing.

\section{Availability of data and materials}

Detailed data produced and analyzed in the current study are available from the corresponding author upon reasonable request.

\section{Authors' contributions}

Tianli Cheng®Conceptualization, Project administration, Writing - Original Draft

Jianfu Heng®Project administration, Writing - Review \& Editing, Formal analysis 
Quanhui Mei『Project administration, Formal analysis

Lijun Chen『Supervision, Writing - Original Draft

Feng Zeng®Conceptualization, Writing - Review \& Editing

\section{References}

1. Lambrecht BN, Hammad H: The immunology of the allergy epidemic and the hygiene hypothesis. Nat Immunol 2017, 18(10):1076-1083.

2. Nelson RK, Bush A, Stokes J, Nair P, Akuthota P: Eosinophilic Asthma. J Allergy Clin Immunol Pract 2020, 8(2):465-473.

3. Qu J, Li Y, Zhong W, Gao P, Hu C: Recent developments in the role of reactive oxygen species in allergic asthma. J Thorac Dis 2017, 9(1):E32-E43.

4. Cevhertas L, Ogulur I, Maurer DJ, Burla D, Ding M, Jansen K, Koch J, Liu C, Ma S, Mitamura Y et al: Advances and recent developments in asthma in 2020. Allergy 2020.

5. Hirose K, Iwata A, Tamachi T, Nakajima H: Allergic airway inflammation: key players beyond the Th2 cell pathway. Immunol Rev 2017, 278(1):145-161.

6. Hough KP, Curtiss ML, Blain TJ, Liu RM, Trevor J, Deshane JS, Thannickal VJ: Airway Remodeling in Asthma. Front Med (Lausanne) $2020,7: 191$.

7. Pfaar O, Alvaro M, Cardona V, Hamelmann E, Mosges R, Kleine-Tebbe J: Clinical trials in allergen immunotherapy: current concepts and future needs. Allergy 2018, 73(9):1775-1783.

8. Lotfi R, Rezaiemanesh A, Mortazavi SH, Karaji AG, Salari F: Immunoresolvents in asthma and allergic diseases: Review and update. J Cell Physiol 2018.

9. Qu J, Do DC, Zhou Y, Luczak E, Mitzner W, Anderson ME, Gao P: Oxidized CaMKII promotes asthma through the activation of mast cells. JCI Insight 2017, 2(1):e90139.

10. Victor JR, Lezmi G, Leite-de-Moraes M: New Insights into Asthma Inflammation: Focus on iNKT, MAIT, and yסT Cells. Clinical Reviews in Allergy \& Immunology 2020.

11. Cai Q, Ye L, Horne R, Bi J, Xu Q, Ye X, Yang A, Jin M, Li X, Lv Q: Patients' adherence-related beliefs about inhaled steroids: application of the Chinese version of the Beliefs about Medicines Questionnaire-specific in patients with asthma. J Asthma 2019:1-8.

12. Cates CJ, Schmidt S, Ferrer M, Sayer B, Waterson S: Inhaled steroids with and without regular salmeterol for asthma: serious adverse events. Cochrane Database Syst Rev 2018, 12:CD006922.

13. Berings M, Karaaslan C, Altunbulakli C, Gevaert P, Akdis M, Bachert C, Akdis CA: Advances and highlights in allergen immunotherapy: On the way to sustained clinical and immunologic tolerance. J Allergy Clin Immunol 2017, 140(5):1250-1267.

14. Bobolea I, Arismendi E, Valero A, Agusti A: Early Life Origins of Asthma: A Review of Potential Effectors. J Investig Allergol Clin Immunol 2019, 29(3):168179.

15. Tay TR, Hew M: Comorbid "treatable traits" in difficult asthma: Current evidence and clinical evaluation. Allergy 2018, 73(7):1369-1382.

16. Galipeau J, Sensebe L: Mesenchymal Stromal Cells: Clinical Challenges and Therapeutic Opportunities. Cell Stem Cell 2018, 22(6):824-833.

17. Giri TK, Alexander A, Agrawal M, Saraf S, Saraf S, Ajazuddin: Current Status of Stem Cell Therapies in Tissue Repair and Regeneration. Curr Stem Cell Res Ther 2019, 14(2):117-126.

18. Pixley JS: Mesenchymal stem cells to treat type 1 diabetes. Biochimica et Biophysica Acta (BBA) - Molecular Basis of Disease 2020, $1866(4): 165315$.

19. Novello S, Debouche A, Philippe M, Naudet F, Jeanne S: Clinical application of mesenchymal stem cells in periodontal regeneration: A systematic review and meta-analysis. J Periodontal Res 2020, 55(1):1-12.

20. Nguyen L, Bang S, Noh I: Tissue Regeneration of Human Mesenchymal Stem Cells on Porous Gelatin Micro-Carriers by Long-Term Dynamic In Vitro Culture. Tissue Engineering and Regenerative Medicine 2019, 16(1):19-28.

21. Quesada MP, García-Bernal D, Pastor D, Estirado A, Blanquer M, García-Hernández AM, Moraleda JM, Martínez S: Safety and Biodistribution of Human Bone Marrow-Derived Mesenchymal Stromal Cells Injected Intrathecally in Non-Obese Diabetic Severe Combined Immunodeficiency Mice: Preclinical Study. Tissue Engineering and Regenerative Medicine 2019, 16(5):525-538.

22. Chen J, Hu C, Chen L, Tang L, Zhu Y, Xu X, Chen L, Gao H, Lu X, Yu L et al: Clinical study of mesenchymal stem cell treating acute respiratory distress syndrome induced by epidemic Influenza A (H7N9) infection, a hint for COVID-19 treatment. Engineering (Beijing) 2020, 6(10):1153-1161.

23. Li Y, Ye Z, Yang W, Zhang Q, Zeng J, Widera D: An Update on the Potential of Mesenchymal Stem Cell Therapy for Cutaneous Diseases. Stem Cells International 2021, 2021:1-18.

24. Nemeth K, Keane-Myers A, Brown JM, Metcalfe DD, Gorham JD, Bundoc VG, Hodges MG, Jelinek I, Madala S, Karpati S et al: Bone marrow stromal cells use TGF-beta to suppress allergic responses in a mouse model of ragweed-induced asthma. Proceedings of the National Academy of Sciences of the United States of America 2010, 107(12):5652-5657.

25. Bonfield TL, Koloze M, Lennon DP, Zuchowski B, Yang SE, Caplan Al: Human mesenchymal stem cells suppress chronic airway inflammation in the murine ovalbumin asthma model. American journal of physiology Lung cellular and molecular physiology 2010, 299(6):L760-770.

26. Fan X-L, Zhang Z, Ma CY, Fu Q-L: Mesenchymal stem cells for inflammatory airway disorders: Promises and challenges. Bioscience Rep 2019.

27. Ou-Yang HF, Huang Y, Hu XB, Wu CG: Suppression of allergic airway inflammation in a mouse model of asthma by exogenous mesenchymal stem cells. Experimental biology and medicine (Maywood, NJ) 2011, 236(12):1461-1467.

28. Zhang LB, He M: Effect of mesenchymal stromal (stem) cell (MSC) transplantation in asthmatic animal models: A systematic review and meta-analysis. Pulmonary pharmacology \& therapeutics 2019(54):39-52. 
29. Silva LHA, Antunes MA, Dos Santos CC, Weiss DJ, Cruz FF, Rocco PRM: Strategies to improve the therapeutic effects of mesenchymal stromal cells in respiratory diseases. Stem Cell Res Ther 2018, 9(1):45.

30. Zhang E, Yang Y, Chen S, Peng C, Lavin MF, Yeo AJ, Li C, Liu X, Guan Y, Du X et al: Bone marrow mesenchymal stromal cells attenuate silica-induced pulmonary fibrosis potentially by attenuating Wnt/beta-catenin signaling in rats. Stem Cell Res Ther 2018, 9(1):311.

31. Seo HA, Moeng S, Sim S, Kuh HJ, Choi SY, Park JK: MicroRNA-Based Combinatorial Cancer Therapy: Effects of MicroRNAs on the Efficacy of Anti-Cancer Therapies. Cells 2019, 9(1).

32. Matsuyama H, Suzuki HI: Systems and Synthetic microRNA Biology: From Biogenesis to Disease Pathogenesis. Int J Mol Sci 2019, 21(1).

33. Tinel C, Lamarthee B, Anglicheau D: MicroRNAs: small molecules, big effects. Curr Opin Organ Transplant 2021, 26(1):10-16.

34. Rodrigo-Munoz JM, Canas JA, Sastre B, Rego N, Greif G, Rial M, Minguez P, Mahillo-Fernandez I, Fernandez-Nieto M, Mora I et al: Asthma diagnosis using integrated analysis of eosinophil microRNAs. Allergy 2018.

35. Pua HH, Happ HC, Gray CJ, Mar DJ, Chiou NT, Hesse LE, Ansel KM: Increased Hematopoietic Extracellular RNAs and Vesicles in the Lung during Allergic Airway Responses. Cell Rep 2019, 26(4):933-944 e934.

36. Bahmer T, Krauss-Etschmann S, Buschmann D, Behrends J, Watz H, Kirsten AM, Pedersen F, Waschki B, Fuchs O, Pfaffl MW et al: RNA-seq-based profiling of extracellular vesicles in plasma reveals a potential role of miR-122-5p in asthma. Allergy 2021, 76(1):366-371.

37. Tang GN, Li CL, Yao Y, Xu ZB, Deng MX, Wang SY, Sun YQ, Shi JB, Fu QL: MicroRNAs Involved in Asthma After Mesenchymal Stem Cells Treatment. Stem cells and development 2016, 25(12):883-896.

38. Qiu L, Zhang Y, Do DC, Ke X, Zhang S, Lambert K, Kumar S, Hu C, Zhou Y, Ishmael FT et al: miR-155 Modulates Cockroach Allergen- and Oxidative StressInduced Cyclooxygenase-2 in Asthma. Journal of immunology 2018, 201(3):916-929.

39. Chen L, Zhong JL: MicroRNA and heme oxygenase-1 in allergic disease. Int Immunopharmacol 2020, 80:106132.

40. Potter ML, Hill WD, Isales CM, Hamrick MW, Fulzele S: MicroRNAs are critical regulators of senescence and aging in mesenchymal stem cells. Bone 2021, 142:115679.

41. Yao Y, Fan XL, Jiang D, Zhang Y, Li X, Xu ZB, Fang SB, Chiu S, Tse HF, Lian Q et al: Connexin 43-Mediated Mitochondrial Transfer of iPSC-MSCs Alleviates Asthma Inflammation. Stem cell reports 2018, 11(5):1120-1135.

42. Lee S, Yu KR, Ryu YS, Oh YS, Hong IS, Kim HS, Lee JY, Kim S, Seo KW, Kang KS: miR-543 and miR-590-3p regulate human mesenchymal stem cell aging via direct targeting of AIMP3/p18. Age (Dordr) 2014, 36(6):9724.

43. Meng F, Henson R, Wehbe-Janek H, Ghoshal K, Jacob ST, Patel T: MicroRNA-21 regulates expression of the PTEN tumor suppressor gene in human hepatocellular cancer. Gastroenterology 2007, 133(2):647-658.

44. Kim RY, Horvat JC, Pinkerton JW, Starkey MR, Essilfie AT, Mayall JR, Nair PM, Hansbro NG, Jones B, Haw TJ et al: MicroRNA-21 drives severe, steroidinsensitive experimental asthma by amplifying phosphoinositide 3-kinase-mediated suppression of histone deacetylase 2. J Allergy Clin Immunol 2017, 139(2):519-532.

45. Lee HY, Lee HY, Choi JY, Hur J, Kim IK, Kim YK, Kang JY, Lee SY: Inhibition of MicroRNA-21 by an antagomir ameliorates allergic inflammation in a mouse model of asthma. Experimental lung research 2017, 43(3):109-119.

46. Dino P, D'Anna C, Sangiorgi C, Di Sano C, Di Vincenzo S, Ferraro M, Pace E: Cigarette smoke extract modulates E-Cadherin, Claudin-1 and miR-21 and promotes cancer invasiveness in human colorectal adenocarcinoma cells. Toxicol Lett 2019, 317:102-109.

47. Chen L, Zhang C, Chen L, Wang X, Xiang B, Wu X, Guo Y, Mou X, Yuan L, Chen B et al: Human Menstrual Blood-Derived Stem Cells Ameliorate Liver Fibrosis in Mice by Targeting Hepatic Stellate Cells via Paracrine Mediators. Stem Cells Transl Med 2017, 6(1):272-284.

48. Cheng T, Hu C, Yang H, Cao L, An J: Transforming growth factor-beta-induced miR143 expression in regulation of non-small cell lung cancer cell viability and invasion capacity in vitro and in vivo. Int J Oncol 2014, 45(5):1977-1988.

49. Chen L, Li Q, Su J, Yang C, Li Y, Rao Y: Trunk kidney of grass carp (Ctenopharyngodon idella) mediates immune responses against GCRV and viral/bacterial PAMPs in vivo and in vitro. Fish Shellfish Immunol 2013, 34(3):909-919.

50. Zeng F, Heng J, Guo X, Wang Y, Wu W, Tang L, Chen M, Wang S, Deng H, Wang J: The novel TP53 3'-end methylation pattern associated with its expression would be a potential biomarker for breast cancer detection. Breast Cancer Res Treat 2020, 180(1):237-245.

51. Knight DA, Rossi FM, Hackett TL: Mesenchymal stem cells for repair of the airway epithelium in asthma. Expert review of respiratory medicine 2010, 4(6):747-758.

52. Wu D, Shi M, Fan XD: Mechanism of miR-21 via Wnt/beta-catenin signaling pathway in human A549 lung cancer cells and Lewis lung carcinoma in mice. Asian Pac J Trop Med 2015, 8(6):479-484.

53. Tang X, Wu F, Fan J, Jin Y, Wang J, Yang G: Posttranscriptional Regulation of Interleukin-33 Expression by MicroRNA-200 in Bronchial Asthma. Molecular therapy : the journal of the American Society of Gene Therapy 2018, 26(7):1808-1817.

54. Lu TX, Munitz A, Rothenberg ME: MicroRNA-21 is up-regulated in allergic airway inflammation and regulates IL-12p35 expression. Journal of immunology 2009, 182(8):4994-5002.

55. Lu TX, Hartner J, Lim EJ, Fabry V, Mingler MK, Cole ET, Orkin SH, Aronow BJ, Rothenberg ME: MicroRNA-21 limits in vivo immune response-mediated activation of the IL-12/IFN-gamma pathway, Th1 polarization, and the severity of delayed-type hypersensitivity. Journal of immunology 2011, 187(6):3362-3373.

56. Gao F, Chiu SM, Motan DA, Zhang Z, Chen L, Ji HL, Tse HF, Fu QL, Lian Q: Mesenchymal stem cells and immunomodulation: current status and future prospects. Cell Death Dis 2016, 7:e2062. 
57. Bernardo Maria E, Fibbe Willem E: Mesenchymal Stromal Cells: Sensors and Switchers of Inflammation. Cell Stem Cell 2013, 13(4):392-402.

58. Yue Q, Zhang Y, Li X, He L, Hu Y, Wang X, Xu X, Shen Y, Zhang H: MiR-124 suppresses the chemotactic migration of rat mesenchymal stem cells toward HGF by downregulating Wnt/beta-catenin signaling. Eur J Cell Biol 2016, 95(9):342-353.

59. Yao L, Zhao H, Tang H, Xiong J, Zhao W, Liu L, Dong H, Zou F, Cai S: Blockade of beta-catenin signaling attenuates toluene diisocyanate-induced experimental asthma. Allergy 2017, 72(4):579-589.

60. Neth P, Ciccarella M, Egea V, Hoelters J, Jochum M, Ries C: Wnt signaling regulates the invasion capacity of human mesenchymal stem cells. Stem Cells 2006, 24(8):1892-1903.

61. Chae WJ, Ehrlich AK, Chan PY, Teixeira AM, Henegariu O, Hao L, Shin JH, Park JH, Tang WH, Kim ST et al: The Wnt Antagonist Dickkopf-1 Promotes Pathological Type 2 Cell-Mediated Inflammation. Immunity 2016, 44(2):246-258.

62. Guo Y, Mishra A, Howland E, Zhao C, Shukla D, Weng T, Liu L: Platelet-derived Wnt antagonist Dickkopf-1 is implicated in ICAM-1/VCAM-1-mediated neutrophilic acute lung inflammation. Blood 2015, 126(19):2220-2229.

63. Ebert R, Benisch P, Krug M, Zeck S, Meissner-Weigl J, Steinert A, Rauner M, Hofbauer L, Jakob F: Acute phase serum amyloid A induces proinflammatory cytokines and mineralization via toll-like receptor 4 in mesenchymal stem cells. Stem Cell Res 2015, 15(1):231-239.

\section{Figures}

\section{Figure 1}

\section{A}
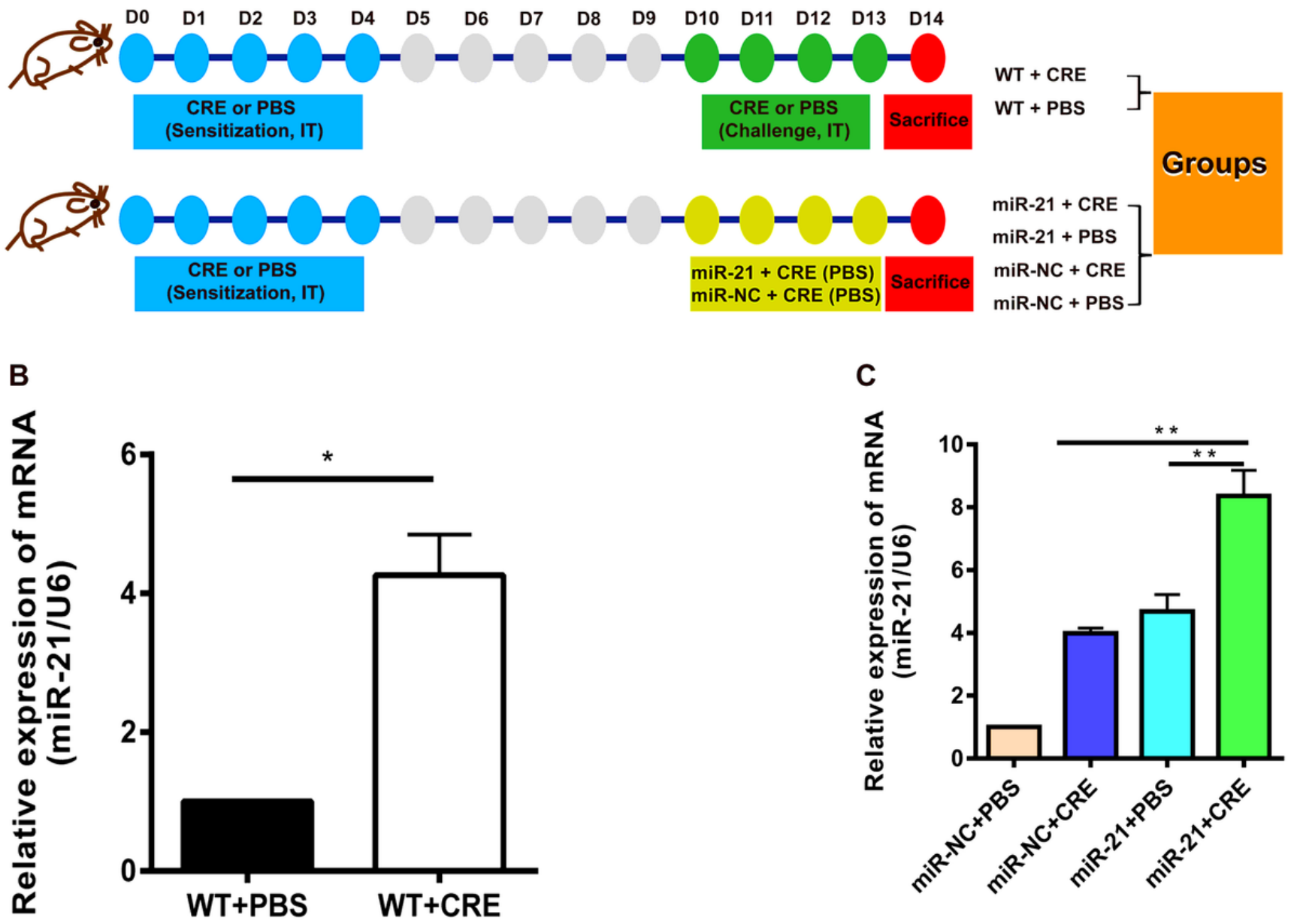

Figure 1

MiR-21 is increased in CRE-induced asthma model mice (A) Protocol for establishment of the CRE-induced asthma mouse model. In the challenge phase, mice were intranasally administered $2 \times 106$ infectious units (IFUs) of lentivirus carrying miR-21 or empty lentivirus. (B) RT-qPCR analysis of miR-21 expression in the lungs of the CRE-challenged asthmatic mice. (C) RT-qPCR analysis of miR-21 expression in the lungs of miR-21- or miR-NC-transfected CRE-challenged asthmatic mice. Data are representative of three independent experiments $(n=6)$. Data are presented as the mean $\pm S E M . * P<0.05,{ }^{*} P<0.01$. 


\section{Figure 2}

A

щ

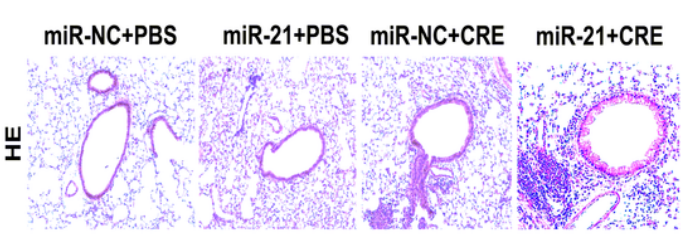

B

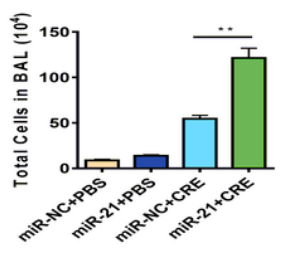

C

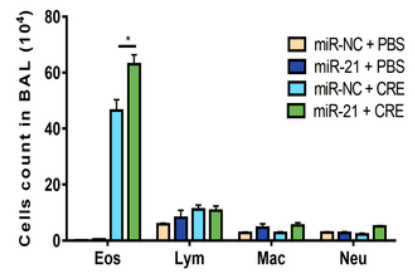

$F$

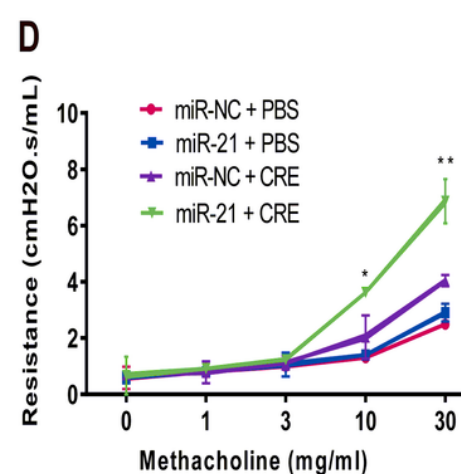

G

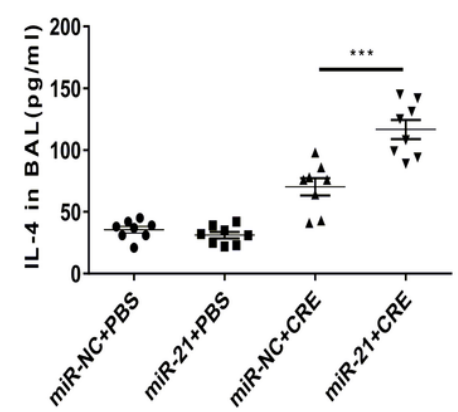

E

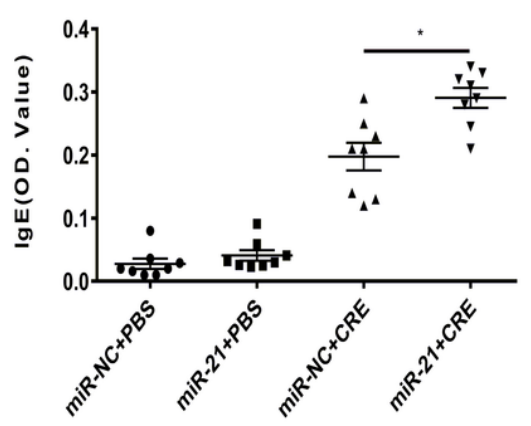

$\mathrm{H}$

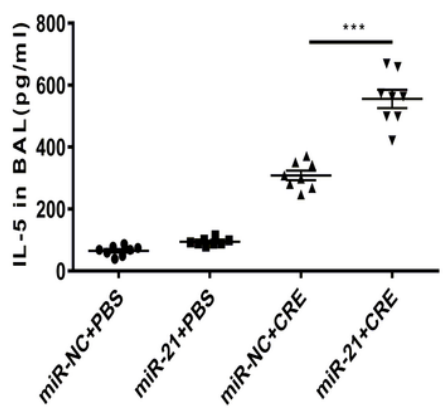

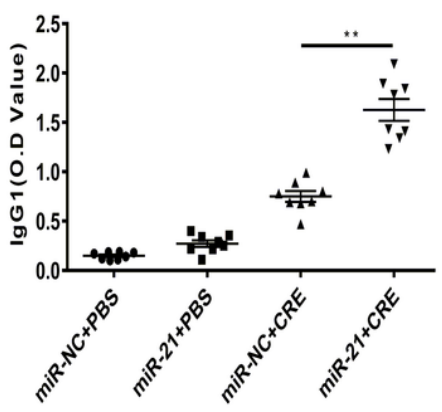

I

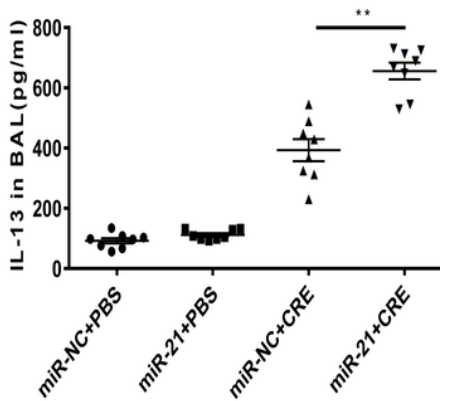

Figure 2

miR-21 promotes lung inflammation in asthma (A) Hematoxylin and eosin (HE) staining was used to evaluated airway inflammation and cell infiltration in PBS- and CRE-treated mice that were transfected with miR-21 or miR-NC. (B) Total cell numbers in BALFs. (C) Numbers of different inflammatory cells (Eos, Lym, Mac, and Neu) in BALFs. (D) Airway hyperresponsiveness to metacholine was analyzed in the different groups. ELISAs were used to evaluate CREspecific IgE (E) and IgG1 (F) levels in BALFs. ELISAs were used to evaluate the Th2 cytokines IL-4 (G), IL-5 (H), and IL-13 (I) in BALFs. Data are representative of three independent experiments $(n=6)$. Data are presented as the mean $\pm S E M$. ${ }^{*} P<0.05,{ }^{\star *} P<0.01, * \star * P<0.001$. 
Figure 3

A
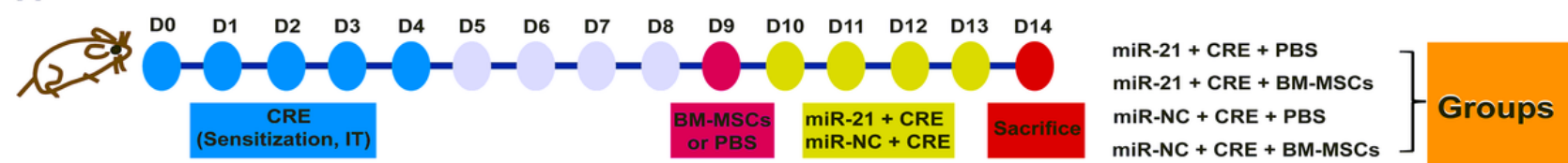

B

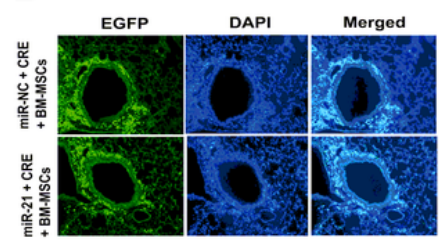

C

D
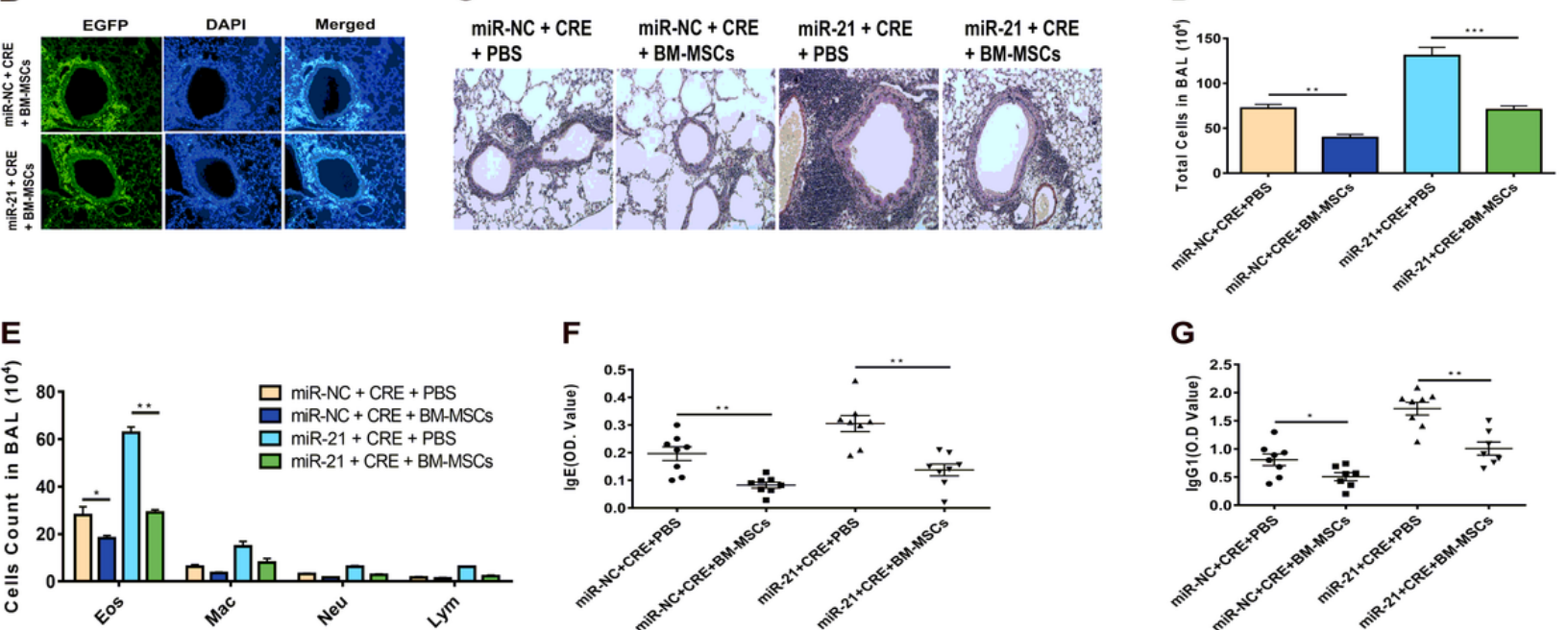

G

H

I
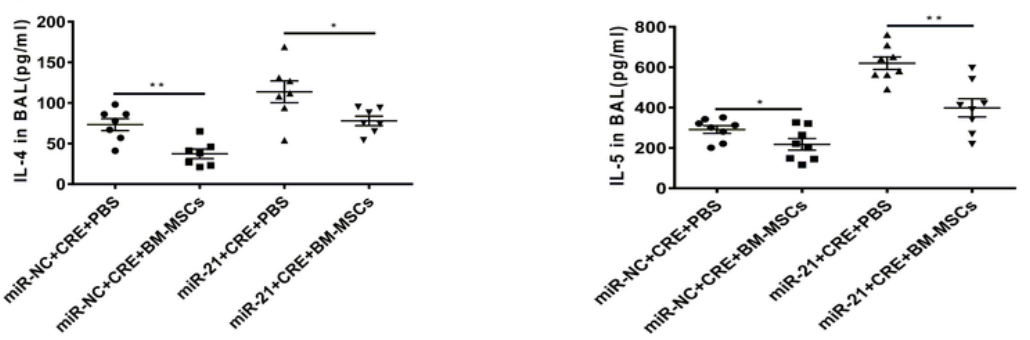

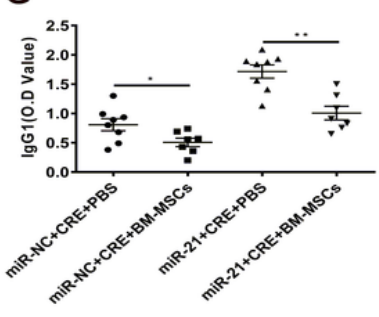

J

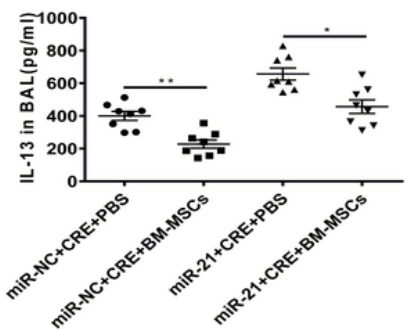

Figure 3

BM-MSCs improve allergic airway inflammation and AHR, whereas miR-21 aggravates the inflammatory reaction by suppressing BM-MSC migration (A) Timeline of BM-MSC engraftment of CRE-induced asthmatic mice. The mice were injected intravenously with BM-MSCs or PBS on 9 day. (B) GFP immunofluorescence was used to demonstrate that BM-MSCs migrated to the lungs. (C) HE staining was used to evaluate airway inflammation infiltration in CRE-induced asthmatic mice treated with BM-MSCs or PBS. Total cell (D) and differentiated leukocyte (E) numbers in BALFs. ELISAs were used to evaluate CRE-specific IgE (F) and IgG1 (G) levels in BALFs. ELISAs were used to evaluate the Th2 cytokines IL-4 (H), IL-5 (I), and IL-13 (J) in BALFs. Data are representative of three independent experiments $(n=6)$. Data are presented as the mean \pm SEM. ${ }^{*} P<0.05,{ }^{\star *} P<0.01,{ }^{\star \star *} P<0.001$. 
Figure 4

A

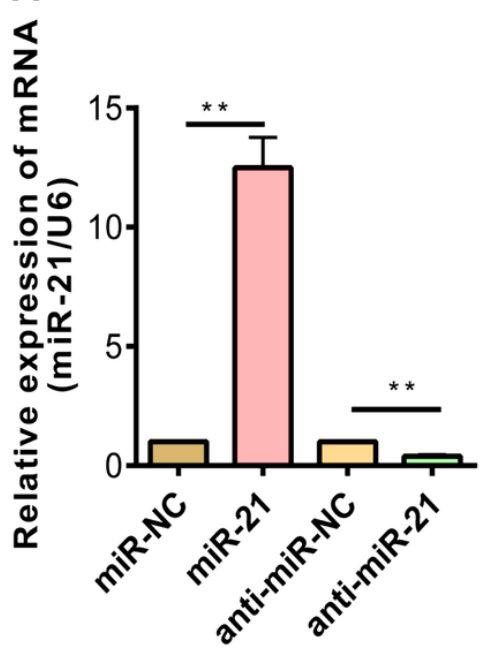

C

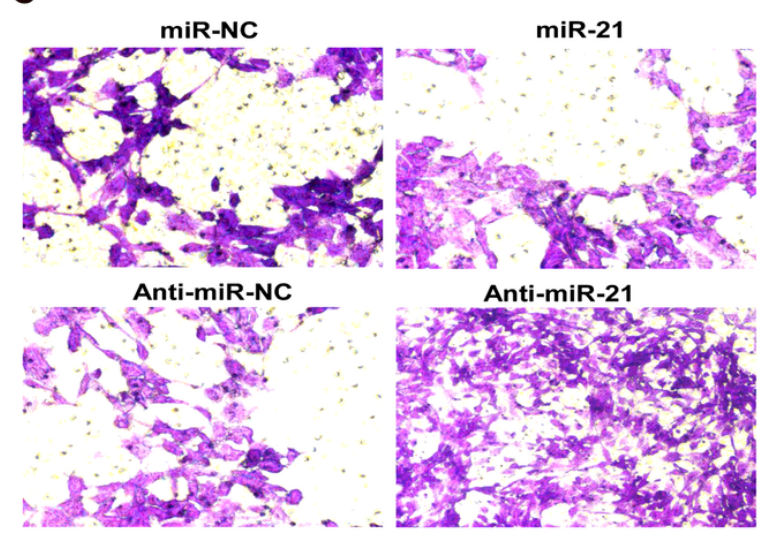

B
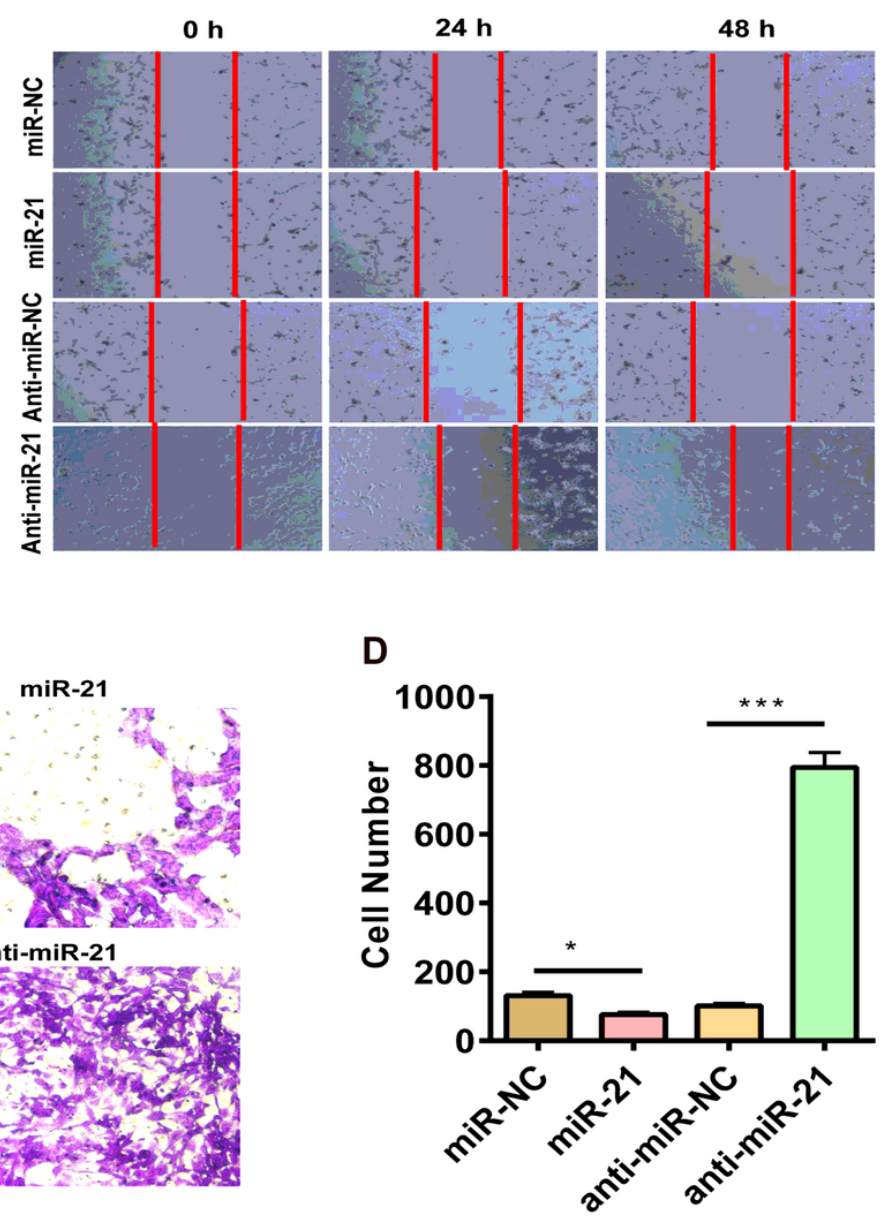

Figure 4

miR-21 suppresses BM-MSC migration in vitro (A) RT-qPCR analysis of miR-21 expression after transfection of BM-MSCs with miR-21, miR-NC, anti-miR-21, or anti-miR-NC. (B) Wound healing assays of BM-MSCs transfected with miR-21, miR-NC, anti-miR-21, or anti-miR-NC. (C) BM-MSCs were transfected with miR21, miR-NC, anti-miR-21, or anti-miR-NC and subjected to Transwell migration assay (magnification, 20x). (D) Quantification of migrated MSCs. Data are representative of three independent experiments. Data are presented as the mean $\pm S E M$. ${ }^{*} P<0.05,{ }^{\star *} P<0.01,{ }^{* \star *} P<0.001$. 
Figure 5

A

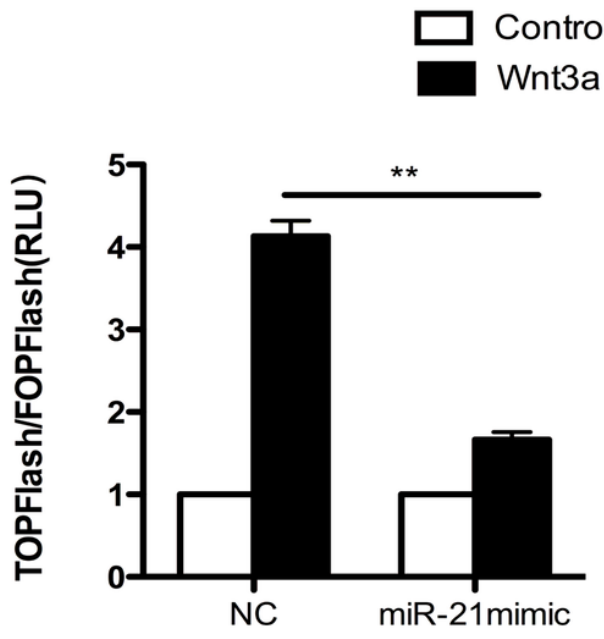

C

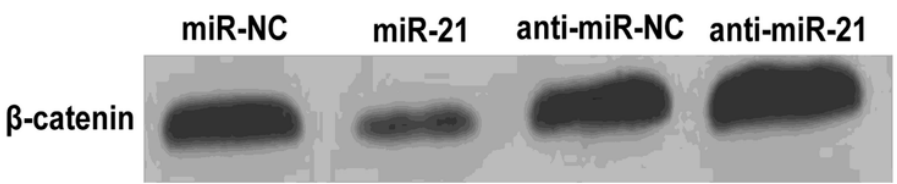

GAPDH

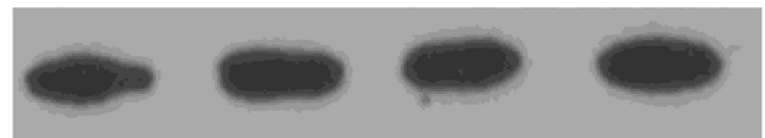

B

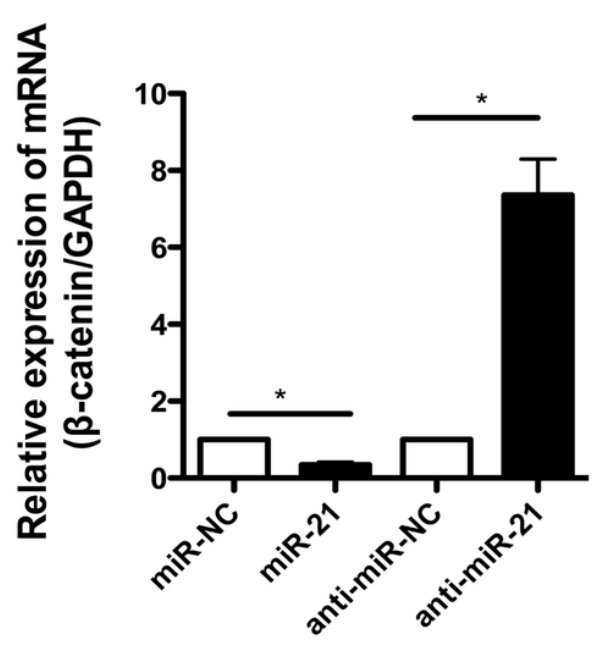

D

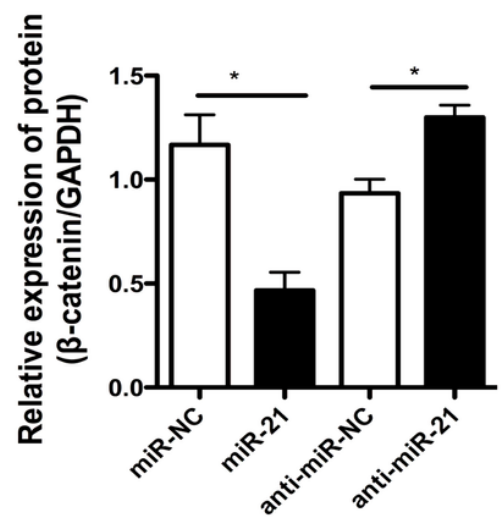

Figure 5

miR-21 inhibits Wnt/ $\beta$-catenin signaling in BM-MSCs (A) TOPFlash/FOPFlash luciferase activity in BM-MSCs treated with miR-21 mimic. (B) RT-qPCR analysis of Ctnnb1 expression in BM-MSCs transfected with miR-21, miR-NC, anti-miR-21, or anti-miR-NC. GAPDH was used as a reference gene. (C) Immunoblotting of $\beta$-catenin in BM-MSCs transfected with miR-21, miR-NC, anti-miR-21, or anti-miR-NC. (D) Protein quantification. All data are reported as the mean \pm SD of three independent experiments. ${ }^{*} \mathrm{P}<0.05$. 
Figure 6

A
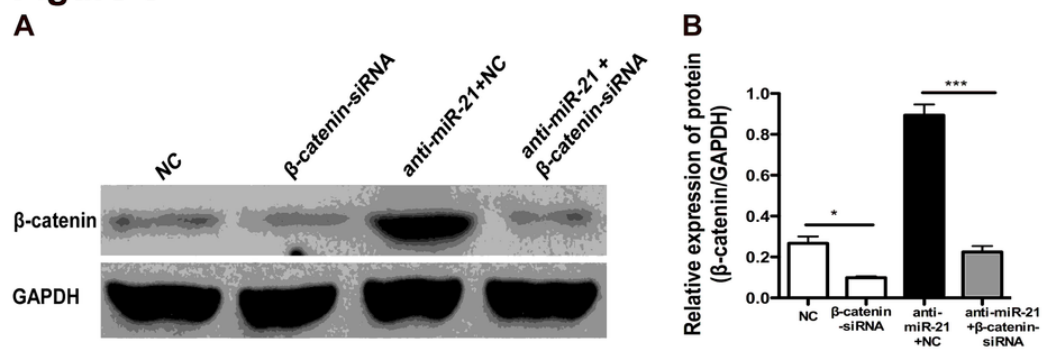

C
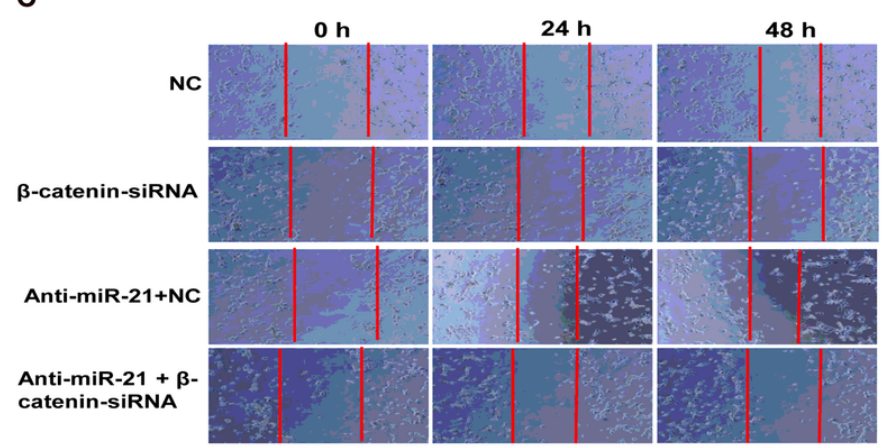

D
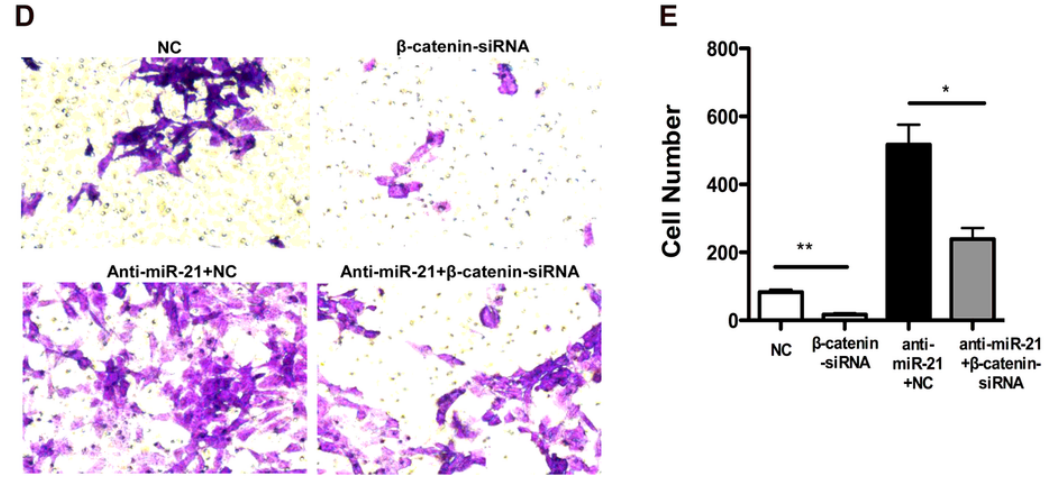

Figure 6

miR-21 suppresses Wnt/ $\beta$-catenin signaling to inhibit BM-MSC migration in vitro (A) $\beta$-Catenin protein in BM-MSCs transfected with anti-miR-21 and $\beta$-cateninsiRNA was detected by western blotting. (B) Protein quantification. (C) Wound healing assays of BM-MSCs transfected with anti-miR-21 and $\beta$-catenin-siRNA. (D) The Boyden chamber assay was used to evaluate the migration ability of BM-MSCs under different treatments. (E) Quantification of migrated cells in the different groups. All data are reported as the mean \pm SD of three independent experiments. ${ }^{*} P<0.05, * * P<0.01, * \star * P<0.001$.

\section{Figure 7}

A

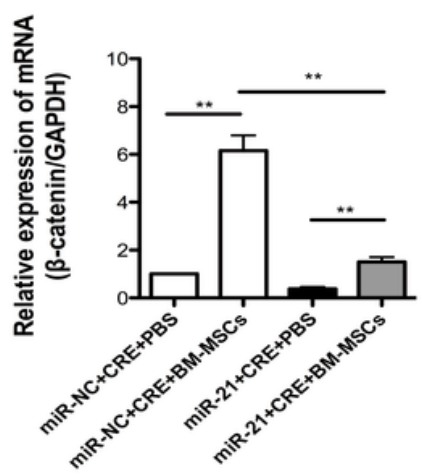

B

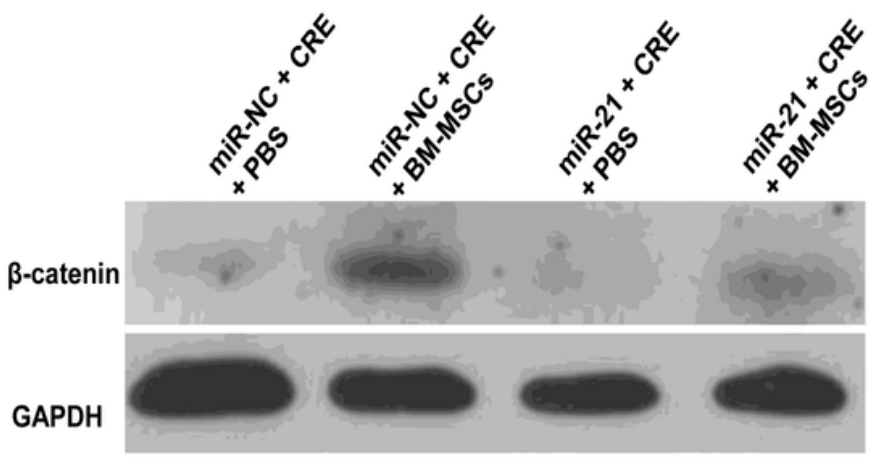

C

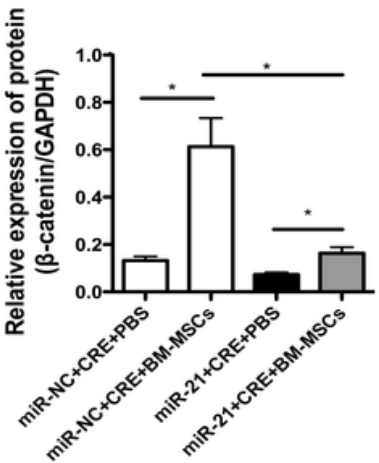

Figure 7

miR-21 inhibits BM-MSC migration by suppressing Wnt/ $\beta$-catenin singling in vivo (A) RT-qPCR analysis of Ctnnb1 mRNA expression in the lungs after transfection of BM-MSCs into CRE-induced asthma model mice. (B) Immunohistochemistry of $\beta$-catenin expression in the lungs of CRE-induced asthma 
model mice. (C) Western blot analysis of $\beta$-catenin protein expression in the lungs of CRE-induced asthma model mice that received miR-21-transfected BMMSCs. (D) Quantification of protein contents. Data are presented as the mean \pm SEM. ${ }^{*} P<0.05, * * * P<0.001$.

Figure 8
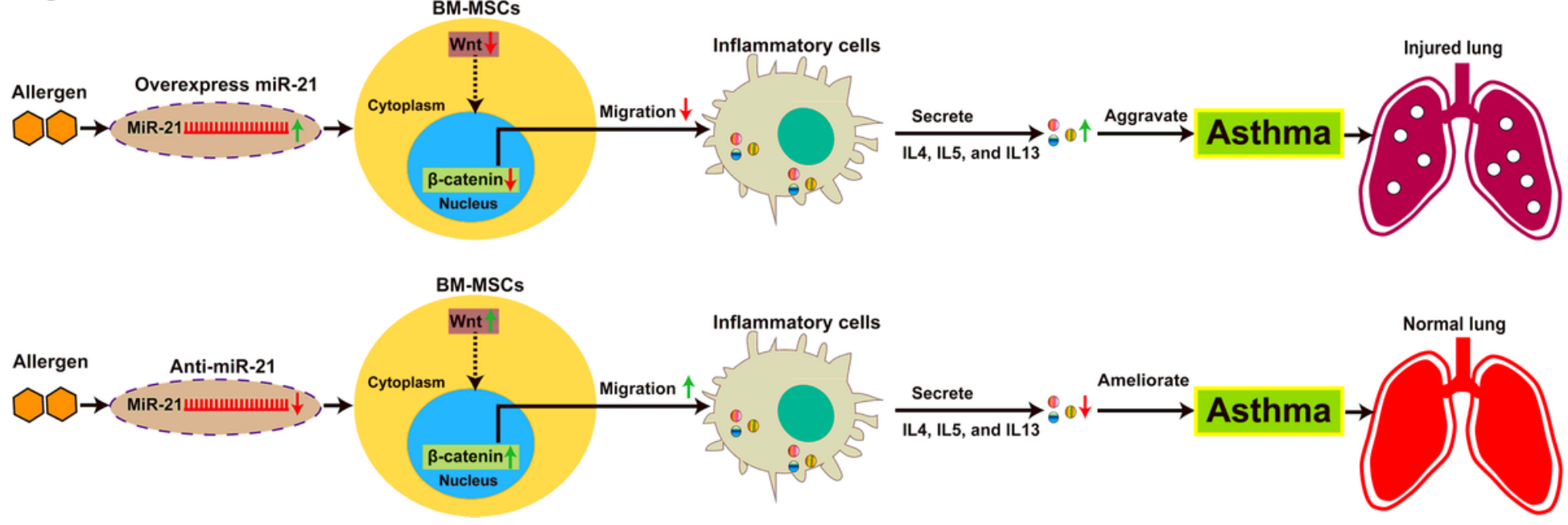

Figure 8

Schematic representation of how miR-21 suppresses MSC migration by suppressing Wnt/ $\beta$-catenin signaling in asthma After allergen invasion, miR-21 expression is significantly increased, and miR-21 downregulates Wnt/ $\beta$-catenin signaling in MSCs. Decreasing Wnt/ $\beta$-catenin signaling suppresses MSC migration. Subsequently, inflammatory cells secrete more TH-2 cytokines (IL-4, IL-5, and IL-13), ultimately resulting in aggravation of asthma development. In contrast, when anti-miR-21 is supplied, a series of signaling cascades will result in increased Wnt/ $\beta$-catenin expression and enhanced MSC migration, thus improving asthma.

\section{Supplementary Files}

This is a list of supplementary files associated with this preprint. Click to download.

- Figures1.tif 